\title{
A cloud-based cognitive computing solution with interoperable applications to counteract illegal dumping in smart cities
}

\author{
Mauro Coccoli ${ }^{1}$ D $\cdot$ Vincenzo De Francesco ${ }^{2} \cdot$ Antonio Fusco $^{3} \cdot$ Paolo Maresca $^{2}$
}

Received: 22 June 2020 / Revised: 19 May 2021 / Accepted: 8 July 2021 /

Published online: 3 September 2021

(c) The Author(s) 2021

\begin{abstract}
The study presented in this paper is the outcome of the activity carried on within the program "Party Cloud Challenge per Genova", promoted by IBM in collaboration with the city municipality of Genoa, Italy. This challenge aimed to show how using cognitive computing solutions in an integrated cloud-based development environment enables the rapid deployment of advanced services with interoperable applications. Specifically, we investigated a solution to cope with the problem of illegal dumping prevention in a smart city. In this respect, we will describe the study of the prototype of an automated visual recognition and alerting system. The presented solution relies on the use of cognitive computing technologies to analyze videos provided by cameras installed in urban areas, to identify trash, especially bulky waste, where it should not be, and trigger an alarm to the municipality. In particular, we want to take advantage of the pictures, frames and videos continuously recorded by cameras installed for traffic monitoring, for surveillance, etc. in smart cities where the waste management system is supposed to be integrated with other municipality services for environment control and management. Besides, an organization plan is also proposed for intelligent waste collection as well as some organizational ideas for scalability.
\end{abstract}

Keywords Smart city $\cdot$ Cloud $\cdot$ Cognitive computing $\cdot$ Image recognition $\cdot$ Waste management $\cdot$ Environment

\section{Introduction}

The possibilities offered by the wide variety of available cloud-based cognitive computing solutions is changing people's lives, as well as the information technology industry methodologies. In fact, the adoption of the cloud computing paradigm in the design, development,

Mauro Coccoli

mauro.coccoli@unige.it

1 DIBRIS, University of Genoa, Genoa, Italy

2 DIETI, Federico II Naples University, Naples, Italy

3 Asia Napoli S.p.a., Naples, Italy 
deploy and delivery of advanced services has created new scenarios where we can easily manage complex, scalable and interoperable systems, obtaining all the required functions "as a service" from a rich catalog [22], in compelling applications. As a test bed to show the effectiveness of this paradigm, we addressed the problem of illegal dumping prevention in a smart city, and we investigated the possibility of developing a smart alerting solution, through the use of the integrated cognitive computing programming environment available within the IBM Cloud platform. This approach allowed using a single orchestrating platform to integrate multiple interoperable applications, with a cloud-native infrastructure support. In fact, the platform easily integrates with existing tools and architectures and is technology agnostic.

The opportunity of integrating easily interoperable applications well fits with the idea of smart city, which is tied to making the most of opportunities provided by novel ICT solutions, deploying mobile intelligent applications to provide effective services seamlessly integrated with the main city infrastructures and sensor networks for environment control and management. Hence, suited solutions should be investigated, exploiting services and data already available and accessible for the development of efficient integrated services. However, the adoption of sophisticated systems and advanced solutions are just enablers for the institutions to set active policies driven to enhance citizens' quality of life and we always must keep in mind that this general view should include $(i)$ technological aspects, (ii) people, and (iii) institutions. In this respect, moving along these three main dimensions, the objectives of a smart city can be summarized as the following: (i) integration of infrastructures and technology-mediated services, (ii) social learning for strengthening human infrastructure, and (iii) governance for institutional improvement and citizens engagement [31]. In this respect, many projects for making a city smart result in the definition of new solutions to mitigate current urban problems, to the aim of improving services to the citizens and, thus, making cities attractive places to live in. From a practical point of view, most smart cities implement a common reference model that addresses global sustainability challenges at a local level [42]. Regardless the implementation details, the available sensors, the installed instrumentations, the adopted interconnection techniques, the integration methodologies, the ways for exchanging data, possibly in open data format, and the number of possible applications, the main topics addressed in designing smart cities services are e-democracy, e-health, sustainable urban mobility, water, energy, environment, pollution.

Applying the smart city paradigm to the field of waste management is a paramount topic for the environment control and management in urban settings and poses challenging issues. Generally speaking, waste management refers to finding solutions and advanced techniques to manage the garbage lifecycle and disposal processes such as, e.g., using sensorized bins in an Internet of Things (IoT) framework [3] or optimizing pickup operations and transports to decentralized dumps. Nevertheless, a smart waste management should embrace an integrated planning strategy tailored for resource recovery and efficiency within a circular economy framework [16]. The more obvious and economical solution is making a call to action, for the people to decrease the quantity and the volume of the rubbish they produce, also encouraging separate collection of waste. However, due to the possible presence of non-polite citizens, we also have to consider policies aimed to the reduction of illegal dumping of bulky waste [15]. A sophisticated way to achieve this objective is using the large amount of data already available within a smart city environment, owing to the sensor networks installed therein. Hence, we regard the smart city as a generator of images, coming from different sources, such as, e.g., the videos collected around by many cameras installed by the municipality and security agencies as well, for purposes other than 
dumping surveillance and we need the seamless integration of data originated from different applications.

Within the general problem of waste management, illegal dumping has huge cost. To have an idea of the importance of this phenomenon, we recall that the environmental deterioration caused by the inefficient management of trash, and specifically of large dimensions waste, is a problem of immediate perception and its social cost must be considered from different perspectives. On the one hand, we have to account the direct costs for the operational management, e.g., actions to prevent the dumping, to perform removals, or to recycle waste. On the other hand, there are indirect costs deriving from the urban environments worsening, in terms of bad perception of quality of life by inhabitants and tourists. For what concerns direct costs, we observe that large dimensions waste represents a problem also for the directly involved workers who must collect them and impacts heavily on the whole costs of management of urban hygiene. Specifically, large dimension waste refers to objects that cannot be hosted in standard trash containers, for both being large and made of different materials that may need special attention to be disassembled before recycling. Usually, this kind of waste is treated through dedicated services for collection on demand, or the citizens can confer their waste in suited collection centers, managed by the municipality. According to the 2019 annual report of the Ispra (Istituto Superiore per la Protezione e la Ricerca Ambientale), the Italian institute for environmental protection and research, the total production of wastes is of 826,076.97 ton/year to be recycled and $134,943.43$ ton/year to be eliminated. On the indirect cost side, we must primarily keep into account the negative impact on tourism, which is the most challenged by this phenomenon. Always with reference to the Italian case, we consider as an example the city of Naples and the Campania region where the data about touristic stream in the period 2008-2018 show a significant drop in 2009, when a serious trash crisis happened. The effect of this drop was comparable to that of the financial crisis occurred in 2013. Then, in 2018, tourism presence in Naples increased to up a $52 \%$ if compared to 2009. In this respect, Istat (Istituto Nazionale di Statistica), the Italian National Statistics Institute, calculated that the economic loss for the city of Naples caused by the trash emergency accounts for about a $6 \%$ of added value for the whole Italian economy, with 65\% coming from internal tourism (about 210 billions of euro) compared to a $35 \%$ of international tourism [18]. In conclusion, in both cases, direct and indirect costs caused by illegal dumping subtract resources that could otherwise be invested in basic services to the community, such as, e.g., welfare, digital infrastructures, urban safety, viability and transports, which represent the basic assets of a modern smart city.

In this flavor, triggered by the online hackathon initiative "IBM Party Cloud Challenge per Genova" (Italian for 'for Genoa'), whose details are described in Sect. 3, this paper focuses on the design of an automated alerting system based on visual recognition, to support the municipality of the city of Genoa, Italy, in counteracting illegal dumping of bulky waste in the streets. Recalling the above-mentioned three-dimensional model, our system implements the following: ( $i$ ) video surveillance systems are integrated with the whole smart city framework and data circulate between interoperable applications; (ii) given the presence of an automated alerting system, citizens are encouraged to not-dumping illegally; (iii) the city government can take specific actions with dedicated prevention policies. In the framework of this hackathon, a prototype centered on the use of cognitive computing technologies and design methodologies was developed, fitting the imposed design constraints. It is worthwhile noticing that the outcome of this activity became part of a bachelor's degree thesis in computer engineering, aimed to show how such technologies can accelerate and improve the learning processes in universities [10-12] and that the thesis was 
awarded for the first prize in the challenge. Hence, this paper has not the ambition of proposing the ultimate system to warn operators when a bulky waste is abandoned outside of authorized disposal points yet introduces a novelty in approaching the design of advanced services through interoperable applications, so that one can integrate third-party and legacy products into an overall solution, while dealing with a variety of standards, interfaces and communication protocols in complex ecosystem environments. This leads to the possibility of developing dynamic, heterogeneous, complex and composed applications that take advantage of the best features from different providers and services simultaneously [33].

The remainder of the paper is structured as follows: Sect. 2 reports related works; Sect. 3 provides an attentive description of the boundaries in which the system was conceived; Sect. 4 presents the proposed smart alerting system and the relevant technical details; results are presented in Sect. 5, also considering the possible impact of the proposed solution in a smart-city framework, which are discussed in Sect. 6. Finally, conclusions and a glance on future works are reported in Sect. 7.

\section{Related works}

Waste management is a continuously evolving topic, which is of paramount importance for the environment. Hence, in this specific field of application, a huge number of studies can be found in the literature, presenting solutions aimed to improve its efficiency. One particular aspect in the complex ecosystem of activities related to waste management, is that of illegal dumping, which can have high social and economic costs, also impacting on territories. Analyzing policies as well as social and economic consequences is out of the scope of this paper, but some hints and references can be found in [15] where waste disposal is examined for Italy, a country which witnesses heterogeneous environmental performances across regions and a decentralized policy system.

From the point of view of the adopted technological solutions, we present a selection of works showing analogies with our case study, which refers to urban settings and, more specifically, smart cities. Even more specifically, it is worthwhile citing once more [16], which refers precisely to the city of Genoa that is our testbed, where Del Borghi et al. developed the "LiguriaCircular" circular economy initiative. The authors highlight that waste management in the smart cities should not only be addressed to operational procedures but also to developing decisional strategies, hence, should be driven by an integrated planning strategy tailored for resource recovery and efficiency in a wider circular economy framework. Their conclusions are based on the analysis of common best practices on smart waste management in Europe, which revealed that ICT applications can enhance intelligent waste management systems, owing to their capabilities of managing data coming from smart cities sensors. Moreover, from their experiences in the field of circular economy, they envisage that the smart city should move towards industrial symbiosis, offering suited tools for regenerative systems and symbiotic business links. Anagnostopoulos et al. [3] consider the opportunities offered by IoT-enabled smart cities, where intelligent services can be provided on top of the information gathered from sensors embedded in the environment to monitor and collect ambient data, also related to aspects of citizens' actions. In this context, the authors present a comprehensive survey of ICT-enabled waste management models, which involve not only the collection of the waste in the field but also the transport and disposal to the appropriate locations. Their finding was that only 6 out of the 32 systems analyzed use IoT-based solutions, leaving space for the definition of new models 
and frameworks for waste management. However, smart city does not only mean sensors and physical systems but also the capability of manipulating the data they produce, with advanced analytics techniques. In the same flavor, Kedia [21] considers the huge quantity of data from 1000s of sensors deployed on top of trash-bins, which can be treated as big data, for they contain varied information on, e.g., capacity, location, time/date of pickup, pickup vehicle assigned, etc. Performing suited analytics, many useful information can be derived, such as, for example, predict exactly when the trash-bins are getting filled and when to schedule pickups so as to avoid higher transportation and fuel cost, labor cost, etc.

All of these papers help us to focus possible waste management scenarios in smart cities and provide a description of the general context we operate in. Then we have concentrated our investigation on systems that use computer vision to recognize and classify garbage. Such systems come from a long tradition of image classification techniques and are used to different aims. For example, Bai et al. [7] present a robotics application for garbage pickup of robots operating on the grass, which allows the robots to detect the garbage accurately and autonomously. The garbage detection mechanism is based on a convolutional neural network $(\mathrm{CNN})$ for recognizing and locating the garbage in images captured by a robot equipped with a Web camera. Moreover, the network can also be used to detect and segment the ground, which is useful for obstacle avoidance. The garbage recognition capability, together with automatic navigation functions, enable the robot to clean garbage on the ground in places like parks or schools efficiently. The test conducted by the authors on their system showed that the garbage recognition accuracy can reach as high as $95 \%$. Always in the field of robotics, Kong et al. [23] present a robot system for intelligent water surface cleaner, whose objective is collecting plastic garbage floating on the water, combining cruise and detection, tracking and steering, and grasping and collection. The real time garbage detection is performed by using the YOLO technique by Redmon et al. [34], which is widely applied in the high speed and accuracy object detection field. The network was trained on a specific floating garbage dataset. Renjith et al. [35] present a fully autonomous robotic device to ensure a hygienic environment for humans to live in. To this aim, they to design and implement a robotic device that collects garbage, recognizing them using a computer vision system based on expeditious background subtraction algorithms, edge and contour detection, in order to obtain accurate results at a high computational speed.

However, garbage classifiers are widely used in many fields, other than robotics application, to support humans and to facilitate manual work. For example, Salmador et al. [36] deal with recycling to preserve the environment and presents an intelligent garbage classifier aimed to analyze images captured by a camera to identify and classify different kinds of objects. The authors used a mixed approach, by combining different techniques. Specifically, they performed three steps: (i) segmentation, to distinguish objects from the background and from other objects through border detection with the Canny algorithm [27]; thresholding, to isolate from the background and eliminate noise; gaussian blur to soften details and filter noise; conversion from color to black and white and binary image using the saturation channel to help find borders; contour detection, (ii) characterization to be able to classify objects by shape, made with two shape descriptors, i.e., Hu moments and Fourier descriptor (see, e.g., [13]), (iii) learning and classification, to classify the characterized objects, comparing them to previously known objects. To this aim a k-Nearest Neighbors classification (k-NN) was used (see, e.g., [29]). As technology changes solutions evolve and garbage classification tasks are now performed with newly available techniques, such as, e.g., in the work by Aral et al. [6], where the authors use deep learning models to classify recyclable garbage. In their study, they look for the most efficient approach testing different well-known models such as, Densenet121, DenseNet169, InceptionResnetV2, 
MobileNet, Xception architectures against the Trashnet dataset. They also compare results achieved by using Adam and Adadelta optimizers. Based on machine learning, Torres et al. [39] introduce another intelligent waste separator, which automatically places waste in different containers by using a multimedia embedded processor and advanced image processing techniques. For the specific case of plastic bottles, Kambam and Aarthi [20] present a sophisticated system to identify different type of plastics for some materials can be recycled, others not. In their approach, the authors used both visual and physical properties to classify the plastic materials. With a suited dataset of plastic objects images, they trained different classifiers to teach them when an object can be recycled or not. Specifically, they used a color-based segmentation algorithm to detect color and a k-NN classifier to predict the color of plastic. In addition, they also used a tactile touch sensor to calculate the pressure that can be applied on the plastic object. Based on the same principles, Anjum and Umar [5] deal with the problem of collecting garbage in residential area through an automated process, which requires the autonomous detection and localization of garbage by the vehicles. In this case, the proposed solution is based on the use of a CNN trained on images labeled as garbage or non-garbage. Another garbage detection application is presented by Lee et al. [24], which introduce a lightweight deep learning model facilitate object detection technology in waste sorting.

Coming to the specific theme of illegal dumping, we observe that many solutions based on visual recognition capabilities were developed. The first research we mention refers to Italy, as our paper does. In a study by Angelino et al. [4], illegal dumps detection is made from multi-temporal satellite images coupled with frequent monitoring inspections of the local environmental agency. Typically, the detection is done via expert photointerpretation in order to achieve a high level of accuracy and reliable maps and this is a time-consuming task. To overcome this problem, the authors implemented a multi-features detection algorithm to classify sites depending on four major characteristics (type, state, location and activity) of the dumps. The automated approach was effective in both finding new illegal spills and to follow the evolution of landfills already found in the past through a multitemporal analysis. A similar work is presented by Vambol et al. [40], where the authors discuss the detection of unauthorized dumps of municipal solid waste using data of remote sensing of Earth and geographic information technologies. Alfarrarjeh et al. [2] present the results obtained from the development of a smart system for monitoring street cleanliness in big cities. In this work, the authors analyze conventional classifiers such as, e.g., Naive Bayes and AdaBoost, which show poor performances. Thus, they propose a geospatial classification approach to enhance the classification accuracy in practice. In particular, they exploit images geo-tagging to devise a novel framework with multiple local trained models exploiting the similarity of local images so that the proposed models learn better street image classification for each geographical region. A case study is presented, based on a large real-world geo-tagged image dataset obtained from Los Angeles Sanitation Department.

Another selection of related works covers applications to detect illegal dumping in smart cities, as the work presented in this paper does. Dabholkar et al. [14] propose a deeplearning-based approach system to override community-based voluntary reporting systems and surveillance-camera-based monitoring systems, which require manual monitoring and are vulnerable to false alarms. Jain et al. [19] deal with the real-time detection of dumping from vehicles by means of frame extraction and its analysis and detection of garbage. In the first step, they use the Haar-cascade classifier method [38] followed by standard background subtraction and frame differencing method. To the same aim, and with the same technique, Mahankali et al. [28] present a system to detect illegal garbage dumping from 
moving vehicles based on video analytics, through the analysis of video sequences of moving vehicles and identifies the owner of the vehicle based on the license plate of the vehicle. Begur et al. [8] focus on illegal dumping in the City of San Jose and proposes a smart mobile-based service system that supports real-time illegal dumping detection, altering, monitoring, and management. The use of surveillance cameras is proposed by Yun et al. [41], within a framework for detecting the unauthorized dumping of garbage by observing the change in relation between persons and the object being held by them through a background subtraction algorithm and human joint estimation. Finally, Mittal et al. [30] propose engaging citizens to track and report on their neighborhoods through a suited smartphone app, which detects and coarsely segments garbage regions in a geo-tagged image. Even in this case the app uses convolutional networks for detecting garbage in images.

To conclude, in Table 1 we made a rough summarization of the main characteristics and functionalities offered by the different solutions illustrated above vs. the ones included in our system.

Table 1 shows that our prototype is based on principles and technologies that are common to the majority of other comparable systems, designed to the aim of solving issues in the same field of application. Moreover, we can find that our prototype combines more features than other ones, which focus on the use of a specific technology. Owing to the adopted development methodology and the tools made available in the IBM Cloud platform, in such an integrated cloud programming environment we could develop a more articulated solution exploiting the SaaS (Software as a Service) and IaaS (Infrastructure as a Service) paradigms to develop interoperable applications.

\section{The 'Challenge per Genova' initiative}

Prior to describing the developed system, to the aim of clarifying the boundaries of the proposed solution, we provide a quick outline of the design specifications that we had to respect. In fact, the solution described in this paper was developed under the regulation of the "IBM Party Cloud Challenge per Genova", which required to develop a prototypal automated visual recognition system to mitigate illegal dumping, using specific tools and services.

In more detail, the "IBM Party Cloud" is an online hackathon organized by IBM in Italy, to the aim of accelerating the technological innovation at the service of sustainability. Specifically, the "Challenge per Genova" track was launched on September 20, 2018 in Milan, lasting November 11, 2018, and it was open to developers, data scientists and IT people in general. This initiative was also supported by Dock Joined in Tech, Lifegate and Codemotion, under the patronage of the Genoa Municipality and its local inhouse garbage company. The objective of the hackathon was that of finding novel solutions to counteract illegal dumping more effectively, especially with reference to the disposal of bulky waste in unauthorized places in the city streets and in urban areas in general. The statement of the problem posed by the challenge is that, presently, most critical situations are spontaneously reported by citizens, even if more than 300 surveillance cameras are installed all over the town. The video surveillance system requires a number of operators to watch videos to find possible abuses and this is not an efficient way of using human resources. Hence, the objective is developing a smart alerting solution able to find in real-time abuses and promptly trigger alarms to the operation center, which can manage timely counteractions to solve issues arisen. 


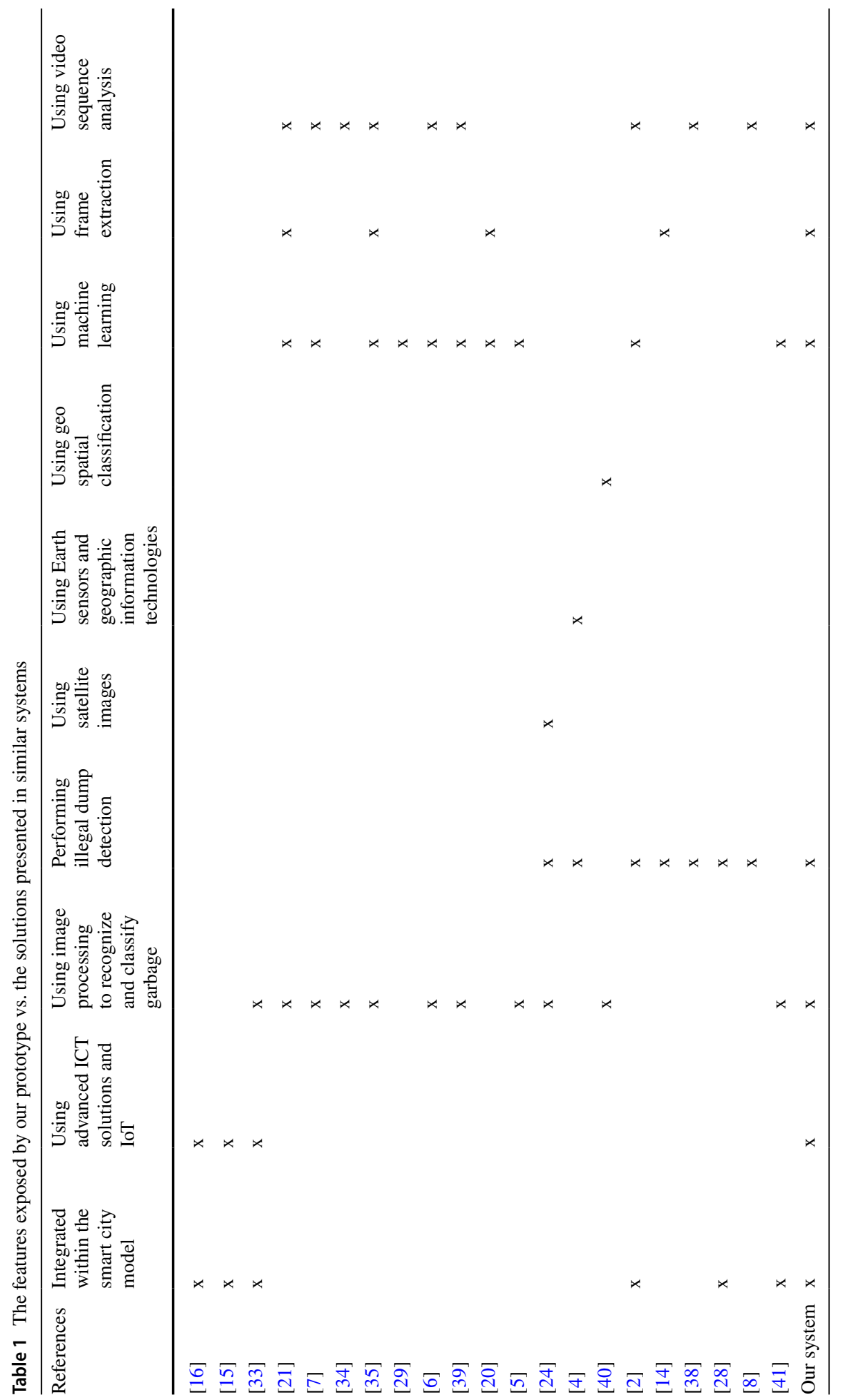


To develop their application prototype, the participants to the contest were provided with full access to the IBM Cloud platform resources and functionalities. In such an integrated environment, designers and developers can exploit advanced image recognition tools and analytics services, which should be used to identify the abuses described above and, consequently, trigger alarms to signal them to operators via SMS, instant messaging systems, or any other means. Even more precisely, any alert is required to include the following information: (i) picture of the evidence, (ii) geo-localization coordinates, (iii) type of bulky waste and its spatial features to evaluate its possible dangerousness, (iv) possible information on "who" made the illegal dumping, e.g., a person as well as a vehicle and its plate number, $(v)$ abnormalities, if any.

After the deadline for submitting the projects, the developed applications were evaluated based on the following criteria: (i) usefulness and value, (ii) bearing on the challenge issue, (iii) technical soundness and accuracy, including architectural blueprints, (iv) interface design and user experience, $(v)$ creativity and novelty. As already said, the solution described in the following was awarded for the first prize.

\section{The smart alerting system}

Given the strict system requirements specified within the framework of the challenge, we programmed an automated alerting system based on visual recognition capabilities, which results in three functional blocks: $(a)$ identification, to detect suspect situations in a specific area under video surveillance, showing possible items of interest. The videos are collected around by the many cameras installed by the municipality and security agencies as well, for purposes other than dumping surveillance. A specific application will find suspect situations and will be the input to our system; (b) classification, to classify objects in the highlighted scenes finding known types of waste; $(c)$ alerting, to warn operators with detailed information and pictures. The architecture of the system is sketched in Fig. 1 where the

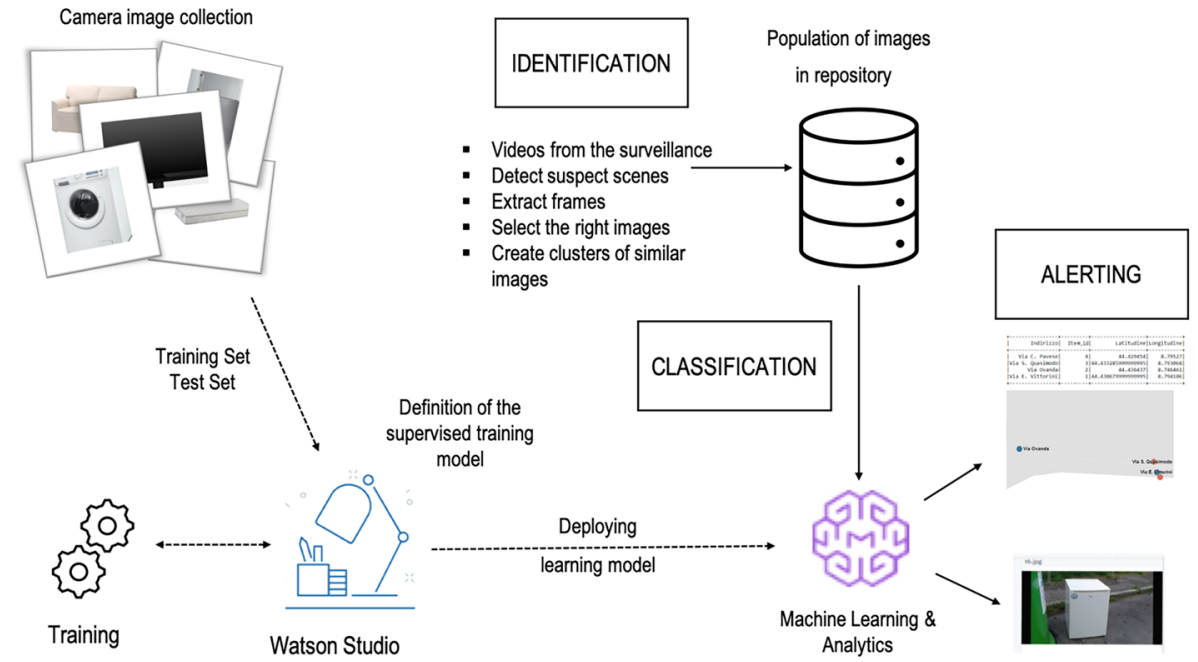

Fig. 1 An overview of the general architecture of the system 
dotted lines are associated to the training activity while solid lines are associated to the application functionalities.

Note that the developed prototype only covers functionalities of "classification" and "alerting", since functions and algorithms of image analysis to detect scenes for the "identification" block are out of the scope of this work and are largely available. In fact, it is easy to find suspect changes in a scene by using consolidated techniques for comparing frames (see, e.g., [32] and references therein), i.e., the stable presence of a new object in the landscape. Also, different techniques exist to identify activities or behaviors in video sequences, in order to detect and isolate specific events. For example, Caruccio et al. [9] present a system for automatic video surveillance, which analyzes record scenes in order to detect the occurrence of specific events defined as targets. For example, let us consider the case of a car in a long-lasting stop and an object candidate to be considered a bulky waste, possibly abandoned by the suspect vehicle. When such frames are isolated and prompted by the video surveillance system, an operator is called to perform a visual analysis of the video, which may lead to the identification of a possible abuse. We want to make this process unattended, implementing an automated visual recognition system able to identify the presence of bulky waste in a given scene and, consequently, trigger alerts. For this reason, we were provided with a selection of videos that were recorded by the local surveillance system of the municipality of Genoa, showing non-fictional scenes of illegal dumping actions of some bulky waste. These images are used to feed the classification system (b) (see details in Sect. 4.1) and, possibly, trigger the alerting system (c) (see details in Sect. 4.2).

All the tools for the development of a software prototype with such functionalities were available within the IBM Cloud platform, which makes all the parts within the system easily interoperable. In more detail, according to the general architecture of the system depicted in Fig. 1, we specify which are the different applications involved. The identification task is performed by an external application, which becomes the input to our system. This feeds the classification system $(b)$, which was developed within the presented prototype exploiting the cognitive computing capabilities offered by IBM Watson. The alerting module $(c)$ also is a service developed within this research and it is realized by using a Jupyter Notebook, available as an open-source web application designed to develop open-source software, open-standards, and services for interactive computing across different programming languages and, among the dozens available, we used Python. For the development of the prototype, we adopted the Watson Studio working environment, which provides suited tools to accelerate infusion of artificial intelligence in applications. Among the array of functionalities offered by IBM Watson, we used: (i) data analysis and visualization, (ii) data preparation and modeling, (iii) machine learning capabilities and visual recognition, (iv) data science tools. The outcome is a system able to classify in real time the bulky waste in unauthorized places and to manage alerts.

\subsection{Visual recognition and classification}

To perform the visual recognition and classification tasks, we used the IBM Watson Visual Recognition service, which offers suited deep learning algorithms to analyze images and to build personalized models so to extract meaning from visual content [1].

As a starting point, we designed our solution based on videos samples and frames, to the aim of programming a customized visual recognition model, able to identify and classify bulky waste in a given area. According to this model (Fig. 1), the supervised learning 
process for the recognition model is crucial and, depending on its accuracy, the application may fail or succeed. Hence, for the custom model to work effectively, an accurate training phase is needed for optimization and to guarantee good performances for the classifier, in terms of precision. Such a process is performed as described in Fig. 2, showing the steps needed for creating and training a specialized visual recognition classifier. More precisely, the following Steps $i-i i i$ are repeated recursively: ( $i)$ prepare training images. Gather files to use as positive and negative data example; (ii) create and train the classifier. Specify the location of the training images and call the Visual Recognition API to create the custom classifier; (iii) test the custom classifier. Classify images with the new custom classifier and measure the classifier performance.

\subsection{Managing alerts}

When in working conditions, the visual recognition system described will be fed with frames extracted at a given sampling time from video surveillance cameras. When a suspect item is identified in at least two consecutive frames of the same scene, an alert is triggered, reporting the class for the suspect item and the relevant confidence score to evaluate the accuracy of the alert. Then, the alert message is completed with metadata attached to the video source, such as a unique video identifier, the timestamp through date and time, the geographical localization through coordinates and street address.

More precisely, the alerts are stored in a database table whose fields are the following (names are reported in English and in the corresponding Italian, when used):

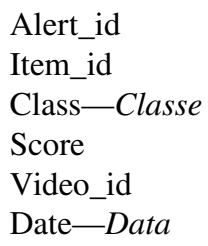

Fig. 2 The recursive steps for training the supervised learning visual recognition classifier

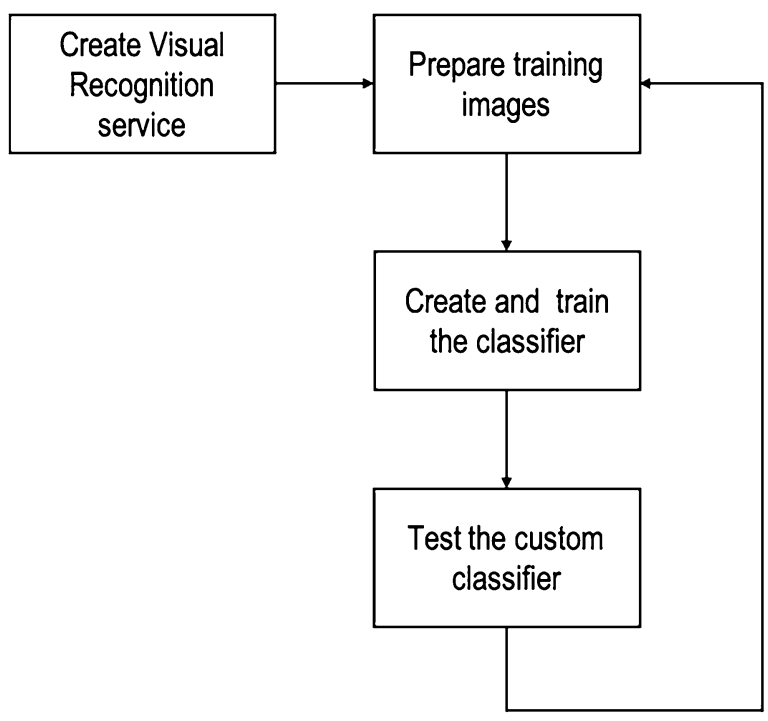


Time-Ora

Latitude-Latitudine

Longitude-Longitudine

Street_address-Indirizzo

When an alert is received the municipality can launch different procedures in reaction, such as, e.g., reporting to another competent authority (e.g., when a license plate is identified in the images and a vehicle has to be investigated) or collecting the waste.

In addition, the data collected within the analysis of the video frames also allow analyzing different aspects. For example, from an operational point of view, we can identify the position and dangerousness of the abandoned object. Other applications are possible, e.g., the analysis of social impact, the subsequent implementation of precise investigation criteria and, finally, in terms of implementing interventions addressed to prevent citizens to adopt such an undesired behavior. To provide an example, the data were processed with a Jupyter Notebook, one of the data science tools included in the Watson Studio, enabling interactive data analysis and visualization. A specific Python Notebook has been programmed to represent the distribution of alerts on a simplified map of Genoa, as shown in Fig. 3.

\section{Results}

We present the currently available results obtained from the tests conducted on the system. Please keep into account that the system was intended as a proof of concept and a demonstrator within the hackathon, not a fully operational service. However, the reported results show that the approach is working fine, and the training phase can be extended with much more classes to further improve performances.

To meet the requirements of the system, we have identified the types of waste that are dumped illegally more often, also matching all the ones included in the sample videos provided by the municipality of Genoa within the Challenge. This resulted in the definition

Fig. 3 Graphical representation of alerts on a map of the city of Genoa

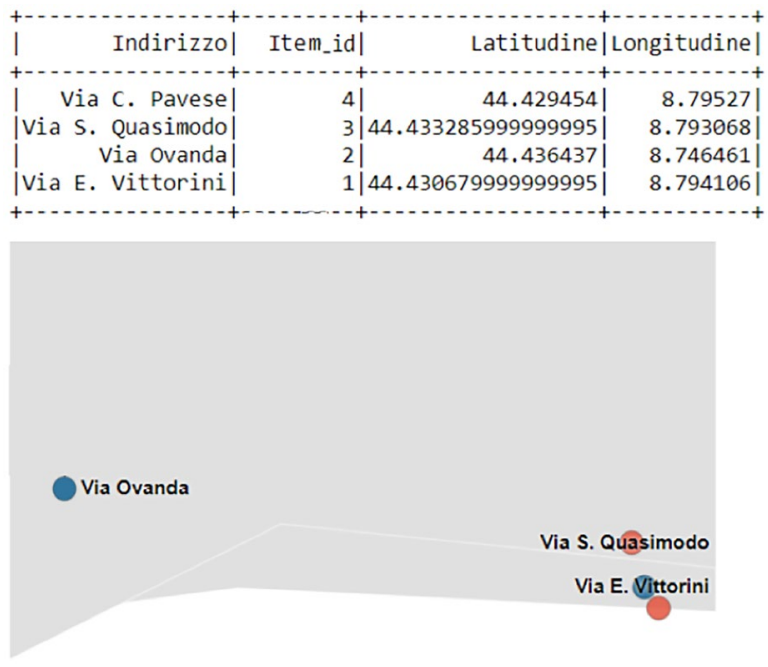


of 13 classes, namely: Refrigerator, Washing machine, Couch, Furniture, Freezer, Bicycle, Armchair, Mattress, Chair, Printer, Television, Bed base, and Heap. In more detail, during the data preparation phase, 35 pictures for each class were considered for training, and 15 pictures for testing. Hence, each class dataset is formed by 50 pictures and the training/test ratio is 70:30. We highlight that the pictures used in the test phase were unrelated with the ones used for the training, so to avoid overfitting. They are the base to compare the custom classificatory accuracy vs. human operators. Then, to mitigate the possible presence of false positive, another class was added, that is the Negative one, for images that do not depict the visual subject of any of the positive classes, thus containing every non-classifiable item such as, e.g., bins on the sidewalk and other scenes containing random objects that should not be associated to any of the considered classes. Accordingly, 35 pictures were used also for this class, against a test set of 15 pictures. It is worthwhile noticing that all the pictures were chosen to fit the holistic approach used by Watson, which consider an image as a whole and cannot discern single elements. For this reason, the pictures used are showing close-up frames of single objects portraited in similar but heterogeneous shots with different values of brightness and contrast, taken from different angles and in varied light conditions. Moreover, to enhance the robustness of the visual recognition system by improving its generalization ability, we modified the color space of the original pictures and applied geometric transformations and filters, to create variants and, thus, enlarging the basic dataset up to 150 pictures per class. A recent survey by Shorten and Khoshgoftaar [37] on image augmentation algorithms discusses many possibilities for doing this, such as geometric transformations, color space augmentations, kernel filters, mixing images, random erasing, feature space augmentation, adversarial training, generative adversarial networks, neural style transfer, and meta-learning.

Figure 4 shows a screenshot taken from the Watson Studio Visual Recognition working environment, presenting the results achieved for some of the considered classes, after the training phase. Please note that the system was programmed in Italian so, for a better

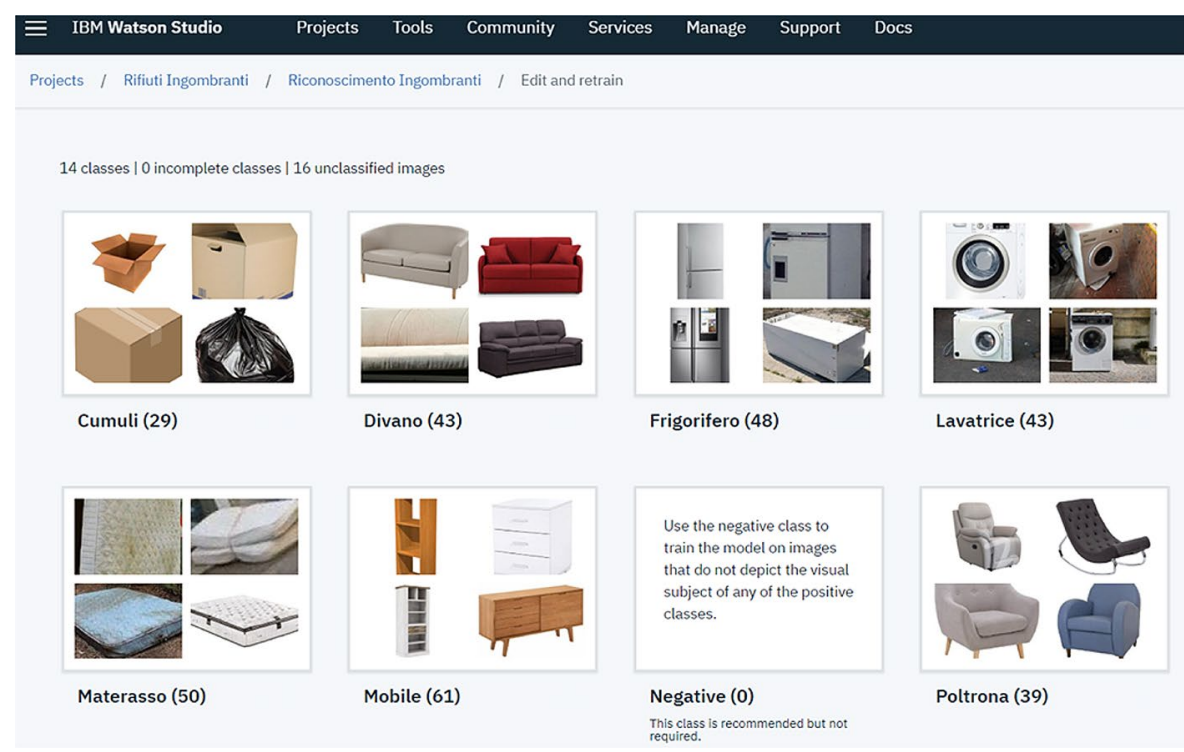

Fig. 4 Results of the tests to evaluate the visual recognition system after its training phase 
Table 2 Italian-English legenda for Fig. 4

\begin{tabular}{llllllll}
\hline Italian & Cumuli & Divano & Frigorifero & Lavatrice & Materasso & Mobile & Poltrona \\
\hline English & Heap & Couch & Refrigerator & Washing machine & Mattress & Furniture & Armchair \\
\hline
\end{tabular}

understanding, consider the Italian-English legenda reported in Table 2 when reading Fig. 4. Also, please note that the numbers in parentheses specify the number of sample pictures associated to each class.

The real effectiveness of the training phase was tested against the frames extracted from the original sample videos, which were provided by the Genoa Municipality, from their files, showing images from real-life scenarios. In addition, we used generic pictures retrieved through common image search engines available over the Web such as, e.g., Google Images, always using the abandoned prefix in the search keys. For each of these pictures, the classifier assigned a matching score to the classes. Please note that such score is a number between 0 and 1, which estimates the model's confidence in the classification, based on the used training set, not an absolute percentage of accuracy. Results are shown in Fig. 5. Accordingly, we conclude that the custom classifier should be considered reliable. In fact, we observe that the pictures are classified with a very high confidence value, i.e., Mattress - 0.86 , Washing machine -0.95 , Freezer -0.90 . This represents a scenario in which the result is reliable and consistent, as the difference between the scores of the classes considered stronger is high. We highlight that we should not think of confidence scores as a measure of absolute truth, rather consider them as a threshold for action. Results with a high confidence score are not consistent and reliable if a false positive is likely. Strong indecision in assigning an image to a class is very rare when the system is able to distinguish the object in the background. When the element is well centered, as test $t 4$ (Washing machine) and test t6 (Freezer) demonstrate,

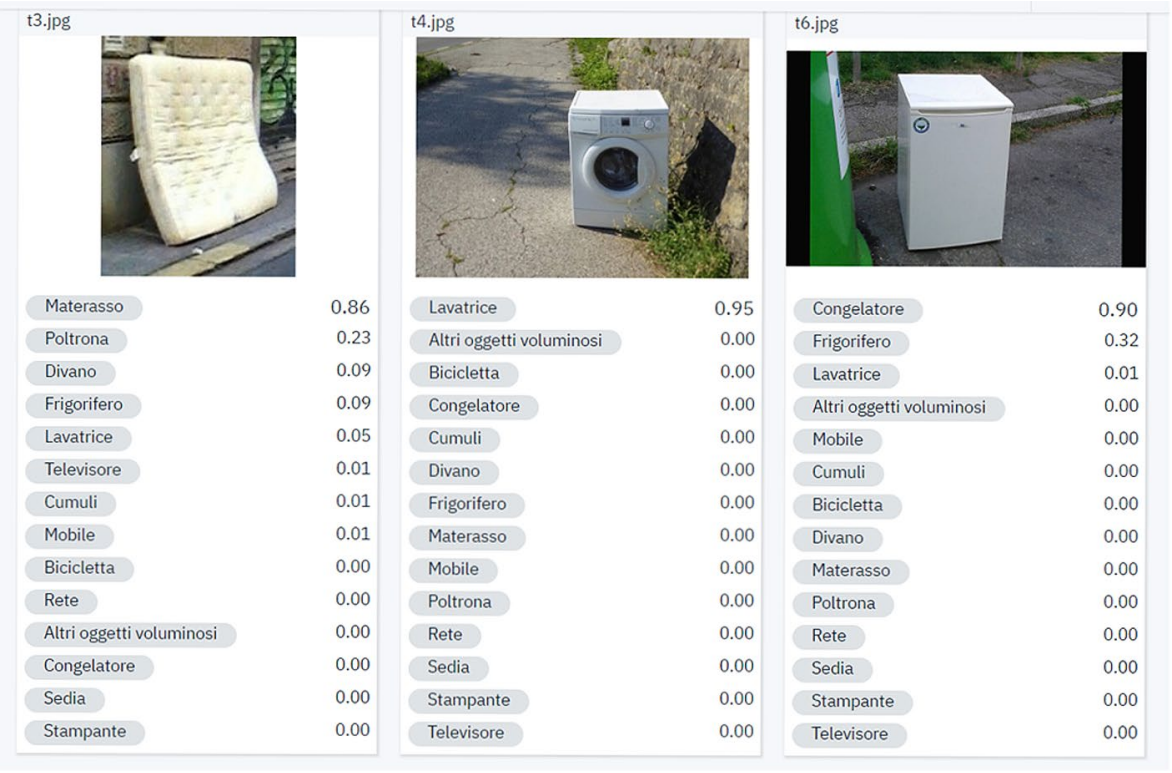

Fig. 5 Results of the test on the trained system 
the confidence score exceeds or equals $90 \%$. In test $\mathrm{t} 3$ (Mattress) the confidence score drops below $90 \%$ but remains an extremely confident and reliable result.

For a better understanding, again, an Italian-English legenda is provided in Table 3.

\section{Discussion}

According to the three dimensional smart-city model already mentioned in the introductory section, which describes a smart city in terms of (i) integration of infrastructures and technology-mediated services, (ii) social learning for strengthening human infrastructure, and (iii) governance for institutional improvement and citizen engagement [31], in the following we report some important considerations relevant to: (i) prospective technology enhancements, (ii) social aspects, (iii) governance.

\subsection{Prospective technology enhancements}

What happens after the system triggers alerts with the relevant information is out of the scope of the developed prototype, which should be duly integrated at the business intelligence level of the existing legacy system through suited interfaces and specific middleware. Moreover, another block in cascade should be able to exploit profitably the information transferred by the classifier, possibly with semantic capabilities. For example, in the case the item identified in a bulky waste dumping event is a TV, it should be associated to a specific hazard class, i.e., the RAEE in Italy, which is not considered in our model, due to limitations imposed by the strict timing and the original requirements of the challenge. This should classify the item on the basis of the subsequent pickup procedure and recovery. Furthermore, other data can drive the decisions. For example, the alert date and its persistency allow going back to the number of days of distress to the community due to the presence of the abandoned object. In this case, the collection system should keep into account a priority order in the organization of the service and logistics by choosing, e.g., the suited vehicle for both the characteristics of the item to be uploaded and the width of the street, that, in Genoa and elsewhere in Italy, can be very narrow. Furthermore, using the geographical coordinates can help calculating optimized routes of the collection paths on the road graph, to plan more efficiently the collection sequence and the overall execution times of the extraordinary withdrawal operations. Future developments should also consider security and privacy issues.

\subsection{Social aspects}

From the point of view of the social impact, the spatial distribution and the identified commodity types allow to construct a dynamic map of the distribution and incidence of the abandonment phenomena, with special attention, e.g., to suburbs. Assuming a principle of proximity at the base of the phenomenon, timely communication and awareness campaigns for users can be started in order to discourage the recurrence of such phenomena. Furthermore, it

Table 3 Italian-English legenda for Fig. 5

\begin{tabular}{llll}
\hline Italian & Materasso & Lavatrice & Congelatore \\
\hline English & Mattress & Washing machine & Freezer \\
\hline
\end{tabular}


is possible to or predict periods of the year in which such illegal dumping phenomena occur more often. This allows planning more frequent controls or other specific actions e.g., with more frequent collection services or making available new cumbersome stuff transfer facilities under a controlled regime, which may be permanent or temporary, i.e., itinerant vehicles.

\subsection{Governance}

Both the above-described aspects are of paramount importance to reduce the impact and the incidence of the phenomena of bulky waste illegal dumping in cities. In fact, the economic and social advantages that may derive from the resolution of this phenomenon are far superior to the costs of activation of specific prevention programs and of a reorganization of existing services. The prototype presented in this paper, contributes first of all to enable a wider understanding of the phenomenon and its causes, on an analytical basis, which may be due to the lack of collaboration of the citizenry and/or the inadequacy of the relevant policies and planned services. In both cases, the availability of a database of reports on a geographical basis, with a precise classification of the abandoned waste, makes it possible to design and test targeted interventions to optimize the resources devoted to solving the problem, very felt by the community in terms of overall perception of the quality of the service provided by the civic operators.

\section{Conclusions and future work}

Following the Challenge per Genova initiative, we faced a specific issue of the waste management problem, which requires more complex solutions and the integration of different interoperable systems. Specifically, we focused our effort on developing and testing an automated visual recognition system based on cognitive computing, and on collecting data and metadata to transmit with alerts, according to the given requirements. As we try expanding the boundaries of the problem analysis, several possibilities arise to extend the basic functionalities of the developed prototype. Depending on the observed field of application, improvements can be done in different directions, to achieve different objectives. In the following we report three examples of possible enhancements:

(i) Expanding the set of data collected, managed and processed, through a suited optimization of the presented visual recognition system. In fact, fully exploiting the cognitive computing capabilities available, we can estimate the overall geometrical characteristics of the detected items both in terms of dimensions (linear and volumetric) and in shape. Moreover, the simultaneous recognition of multiple objects in a single frame or at least the identification of the prevailing commodity fraction would be a desirable feature, which could be achieved exploiting YOLO-based techniques (see, e.g., [17, 25, 26] and references therein), also available in the IBM Cloud Platform, thus easily interoperable with our system. With such information available, alerts would include the GPS coordinates of the interested place as well as the expected number of objects to be collected there, including their type, size, and possible dangerousness;

(ii) Optimizing the collection path design, based on the information provided by the alerts for any point an abandoned item is detected in. A possible solution is performing a classification for the collection points, according to the gravity of the situation 
and the numerosity of waste and their dimensions and expected weight; as well as for the time spent from the alert. This can result in a priority-based list of sites to be visited and give hints on the best suited vehicle for safe collection and transport operations;

(iii) Creating an interactive reporting service so that users, i.e., citizens, can signal abuses, taking pictures and uploading them with data such as, e.g., a description of the object, precise position with address, other useful information, including personal ones. The same interface can also be exploited to prevent illegal dumping by implementing a pick-up service on-demand, in the case a cumbersome object has to be trashed by any citizen.

Acknowledgements The research has been conducted in the metropolitan area of the city of Genoa, Italy, within the program "Party Cloud Challenge per Genova" promoted by IBM Italia, Dock Joined in Tech, Lifegate and Codemotion, under the patronage of the Genoa Municipality and AMIU, the local inhouse garbage company.

Funding Open access funding provided by Università degli Studi di Genova within the CRUI-CARE Agreement.

Open Access This article is licensed under a Creative Commons Attribution 4.0 International License, which permits use, sharing, adaptation, distribution and reproduction in any medium or format, as long as you give appropriate credit to the original author(s) and the source, provide a link to the Creative Commons licence, and indicate if changes were made. The images or other third party material in this article are included in the article's Creative Commons licence, unless indicated otherwise in a credit line to the material. If material is not included in the article's Creative Commons licence and your intended use is not permitted by statutory regulation or exceeds the permitted use, you will need to obtain permission directly from the copyright holder. To view a copy of this licence, visit http://creativecommons.org/licenses/by/4.0/.

\section{References}

1. Ahmed I, House R, Deilma N, Luo L (2019) Custom visual recognition model with Watson studio. Proceedings of the 29th Annual International conference on computer science and software engineering. CASCON, Yarmouth, pp 376-377

2. Alfarrarjeh A, Kim SH, Agrawal S, Ashok M, Kim SY, Shahabi C (2018) Image classification to determine the level of street cleanliness: a case study. Proceedings of the 2018 IEEE Fourth International conference on multimedia big data (BigMM). IEEE, Xi'an, pp 1-5

3. Anagnostopoulos T, Zaslavsky A, Kolomvatsos K, Medvedev A, Amirian P, Morley J, Hadjieftymiades $S$ (2017) Challenges and opportunities of waste management in IoT-enabled smart cities: a survey. IEEE Trans Sustain Comput 2(3):275-289

4. Angelino CV, Focareta M, Parrilli S, Cicala L, Piacquadio G, Meoli G, De Mizio M (2018) A case study on the detection of illegal dumps with GIS and remote sensing images. In: Proceedings of SPIE 10790, Earth Resources and Environmental Remote Sensing/GIS Applications

5. Anjum M, Umar MS (2018) Garbage localization based on weakly supervised learning in Deep Convolutional Neural Network. Proceedings of the 2018 International conference on advances in computing, communication control and networking (ICACCCN). IEEE, Greater Noida, pp 1108-1113

6. Aral RA, Keskin ŞR, Kaya M, Hacıömeroğlu M (2018) Classification of trashnet dataset based on deep learning models. Proceedings of the 2018 IEEE International Conference on Big Data (Big Data). IEEE, Seattle, pp 2058-2062

7. Bai J, Lian S, Liu Z, Wang K, Liu D (2018) Deep learning based robot for automatically picking up garbage on the grass. Proc IEEE Trans Consum Electron 64(3):382-389

8. Begur H, Dhawade M, Gaur N, Dureja P, Gao J, Mahmoud M, Huang J, Chen S, Ding X (2017) An edge-based smart mobile service system for illegal dumping detection and monitoring in San Jose. Proceedings of the 2017 IEEE SmartWorld, ubiquitous intelligence \& computing, advanced \& trusted 
computed, scalable computing \& communications, cloud \& big data computing, Internet of People and Smart City Innovation. IEEE, San Francisco, pp 1-6

9. Caruccio L, Polese G, Tortora G, Iannone D (2019) EDCAR: a knowledge representation framework to enhance automatic video surveillance. Expert Syst Appl 131:190-207

10. Coccoli M, Maresca P, Stanganelli L, Guercio A (2015) An experience of collaboration using a PaaS for the smarter university model. J Vis Lang Comput 31:275-282

11. Coccoli M, Maresca P, Stanganelli L (2016) Cognitive computing in education. J e-Learn Knowl Soc 12(2):55-69

12. Coccoli M, Maresca P, Stanganelli L (2017) The role of big data and cognitive computing in the learning process. J Vis Lang Comput 38:97-103

13. Conseil S, Bourennane S, Martin L (2007) Comparison of Fourier descriptors and Hu moments for hand posture recognition. Proceedings of the 2007 15th European signal processing conference. IEEE, Poznan, pp 1960-1964

14. Dabholkar A, Muthiyan B, Srinivasan S, Ravi S, Jeon H, Gao J (2017) Smart illegal dumping detection. Proceedings of the 2017 IEEE Third International conference on big data computing service and applications (BigDataService). IEEE, San Francisco, pp 255-260

15. D'Amato A, Mazzanti M, Nicolli F, Zoli M (2018) Illegal waste disposal: enforcement actions and decentralized environmental policy. Socioecon Plann Sci 64:56-65

16. Del Borghi A, Gallo M, Strazza C, Magrassi F, Castagna M (2014) Waste management in smart cities: the application of circular economy in Genoa (Italy). Impresa Progetto Electron J Manag $4: 1-13$

17. Huang J, Rathod V, Sun C, Zhu M, Korattikara A, Fathi A, Murphy K (2017) Speed/accuracy trade-offs for modern convolutional object detectors. Proceedings of the IEEE conference on computer vision and pattern recognition. IEEE, Piscataway, pp 7310-7311

18. Istat (2020) Conto satellite del turismo per l'Italia (in Italian), Istat, Istituto Nazionale di Statistica https://www.istat.it/it/archivio/244487. Accessed 26 Mar, 2021

19. Jain S, Gupta N, Khanna A, Gupta A, Gupta D (2020) Detection of garbage disposal from a mobile vehicle using image processing. Proceedings of the International conference on innovative computing and communications. Springer, Singapore, pp 699-709

20. Kambam LR, Aarthi R (2019) Classification of plastic bottles based on visual and physical features for waste management. Proceedings of the 2019 IEEE International conference on electrical, computer and communication technologies (ICECCT). IEEE, Coimbatore, pp 1-6

21. Kedia P (2016) Big data analytics for efficient waste management. Int $\mathrm{J}$ Res Eng Technol 5(10):208-211

22. Kochut A, Deng Y, Head MR, Munson J, Sailer A, Shaikh H, Tang C, Amies A, Beaton M, Geiss D, Herman D, Macho H, Pappe S, Peddle S, Rendahl R, Tomala Reyes AE, Sluiman H, Snitzer B, Volin T, Wagner H (2011) Evolution of the IBM cloud: enabling an enterprise cloud services ecosystem. IBM J Res Dev 55(6):1-13

23. Kong S, Tian M, Qiu C, Wu Z, Yu J (2019) IWSCR: an intelligent water surface cleaner robot for collecting floating garbage. IEEE Trans Syst Man Cybern Syst. https://doi.org/10.1109/TSMC. 2019.2961687

24. Lee S-H, Yeh C-H, HouT-W Y-S (2019) A lightweight neural network based on AlexNet-SSD model for garbage detection. Proceedings of the 2019 3rd high performance computing and cluster technologies conference (HPCCT 2019). Association for computing machinery. HPCCT, New York, pp 274-278

25. Lin TY, Maire M, Belongie S, Hays J, Perona P, Ramanan D, Zitnick CL (2014) Microsoft coco: common objects in context. Proccedings of the European conference on computer vision. Springer, Cham, pp 740-755

26. Liu W, Anguelov D, Erhan D, Szegedy C, Reed S, Fu CY, Berg AC (2016) Ssd: single shot multibox detector. Proceedings of the European conference on computer vision. Springer, Cham, pp 21-37

27. Luo Y, Duraiswami R (2008) Canny edge detection on NVIDIA CUDA. Proceedings of the 2008 IEEE computer society conference on computer vision and pattern recognition workshops. IEEE, Anchorage, pp 1-8

28. Mahankali S, Kabbin SV, Nidagundi S, Srinath R (2018) Identification of illegal garbage dumping with video analytics. Proceedings of the 2018 International Conference on advances in computing, communications and informatics (ICACCI). IEEE, Bangalore, pp 2403-2407

29. Manocha S, Girolami MA (2007) An empirical analysis of the probabilistic K-nearest neighbour classifier. Pattern Recogn Lett 28(13):1818-1824

30. Mittal G, Yagnik KB, Garg M, Krishnan NC (2016) SpotGarbage: smartphone app to detect garbage using deep learning. Proceedings of the 2016 ACM International joint Conference on 
pervasive and ubiquitous computing (UbiComp'16). Association for computing machinery. IEEE, New York, pp 940-945

31. Nam T, Pardo TA (2011) Conceptualizing smart city with dimensions of technology, people, and institutions. Proceedings of the 12th Annual International conference on digital government research: digital government innovation in challenging times. DG-O, College Park, pp 282-291

32. Ng HF, Chin CY (2019) Effective scene change detection in complex environments. Int J Comput Vision Robot 9(3):310-328

33. Nogueira E, Moreira A, Lucrédio D, Garcia V, Fortes R (2016) Issues on developing interoperable cloud applications: definitions, concepts, approaches, requirements, characteristics and evaluation models. J Softw Eng Res Dev 4(7):1-23

34. Redmon J, Divvala S, Girshick R, Farhadi A (2016) You only look once: unified, real-time object detection. Proceedings of the IEEE conference on computer vision and pattern recognition. IEEE, Piscataway, pp 779-788

35. Renjith R, Reshma R (2019) Design and implementation of automatic garbage collecting robot using background subtraction based computer vision algorithm. Proceedings of the 2019 9th International Symposium on Embedded Computing and System Design (ISED). IEEE, Kollam, pp 1-5

36. Salmador A, Pérez Cid J, Rodríguez Novelle I (2008) Intelligent garbage classifier. Int J Interact Multimed Artif Intell (IJIMAI) 1(1):31-36

37. Shorten C, Khoshgoftaar TM (2019) A survey on image data augmentation for deep learning. J Big Data 6(60):12. https://doi.org/10.1186/s40537-019-0197-0

38. Soo S (2014) Object detection using Haar-cascade classifier. Institute of Computer Science, University of Tartu, Tartu, pp 1-12

39. Torres-García A, Rodea-Aragón O, Longoria-Gandara O, Sánchez-García F, González-Jiménez LE (2015) Intelligent waste separator. Comput Sist 19(3):487-500

40. Vambol S, Vambol V, Sundararajan M, Ansari I (2019) The nature and detection of unauthorized waste dump sites using remote sensing. Ecol Quest 30(3):43-55

41. Yun K, Kwon Y, Oh S, Moon J, Park J (2019) Vision-based garbage dumping action detection for realworld surveillance platform. ETRI J 41:494-505

42. Zygiaris S (2013) Smart city reference model: assisting planners to conceptualize the building of smart city innovation ecosystems. J Knowl Econ 4:217-231

Publisher's Note Springer Nature remains neutral with regard to jurisdictional claims in published maps and institutional affiliations. 